\title{
Imaging of neuroinflammation
}

\author{
Andrea Ciarmiello
}

Published online: 14 October 2011

(C) Springer-Verlag 2011

For those of us interested in molecular imaging of the major neurodegenerative disorders, the last decade has witnessed a marked advancement. From basic research to clinical studies, the field has evolved using multidisciplinary techniques and skills, leading to results of significant clinical interest. Reasoning on the role of inflammation in neurodegenerative disease, the recent paper by Santillo et al. [1] is an example of the breadth and depth of the evolving impact of molecular imaging on the field of neurodegeneration and stimulates a reflection on the state of the art of target-specific positron emission tomography (PET) probes of inflammation for brain imaging.

\section{Inflammation in neurodegenerative disease}

Reactive astrogliosis is a ubiquitous hallmark of all central nervous system (CNS) pathologies. Chronic neuroinflammation is associated with a broad range of neurodegenerative diseases, including Alzheimer's disease (AD), Parkinson's disease (PD), amyotrophic lateral sclerosis (ALS), spinal muscular atrophy (SMA), Huntington's disease (HD) and all of the tauopathies [2]. Although the key of molecular and cellular events underlying development of different neurodegenerative disorders is clearly divergent, it should be pointed out that death of neurons depends on activation of resident microglial populations in specific brain regions. Up to now some of the molecular and cellular mechanisms of neurodegeneration have been clearly identified.

Neurodegenerative diseases are often characterized by intraneuronal as well as extracellular accumulation of fibrillary materials. Formation of intracellular inclusion bodies resulting

A. Ciarmiello $(\bowtie)$

Nuclear Medicine, S. Andrea Hospital,

Via V. Veneto 197,

19100 La Spezia, Italy

e-mail: andrea.ciarmiello@as15.liguria.it from aberrant protein folding, abnormal protein-protein interactions and/or dysregulation of the ubiquitin-proteasome system (UPS) are thought to play a principal role in neuronal dysfunction and death of neurons that characterizes several common neurodegenerative diseases [3].

Brain-resident macrophages (microglia) activation plays a critical role in normal brain function by mediating innate immune responses and are the primary mediators of neuroinflammatory responses. Astrocytes, highly differentiated cells widely distributed in the entire CNS, contribute to every major aspect of brain development, function and disease such as regulation of cerebral blood flow and maintenance of synaptic function, neuronal metabolism and neurotransmitter synthesis [4]. Along with other glial cells such as oligodendrocytes and microglia, astrocytes respond to all forms of CNS insults such as infection, trauma and ischaemia by a process commonly referred to as reactive astrogliosis, which involves changes in their molecular expression and morphology [2]. The growing body of evidence on the role inflammation in neurodegenerative diseases stimulated the demand for imaging modalities for the evaluation of inflammation in human brain, which is the topic of this editorial "Imaging of neuroinflammation". Some specific proinflammatory factors have been brought to the attention of scientists as possible targets for molecular imaging probes.

\section{Translocator protein system}

Translocator protein (TSPO) previously known as the peripheral benzodiazepine receptor is an $18 \mathrm{kDa}$ protein located on the outer membrane of mitochondria overexpressed by activated microglia. TSPO is directly or indirectly involved in many functions, including regulation of cholesterol transport [5], synthesis of steroid hormones [6] and apoptosis [7].

TSPO is expressed only at low levels in the healthy human brain. Increased expression has been observed using the 
specific ligand ${ }^{11} \mathrm{C}-\mathrm{PK} 11195$, which has been shown to reflect the distribution of activated microglia and used as a marker transition from a resting to an activated state in experimental studies and human brain disease [8]. Increased brain binding of ${ }^{11} \mathrm{C}$-PK11195 was observed in several acute neurological and neurodegenerative disorders such as strokeinduced brain injury, multiple sclerosis, glioma, refractory epilepsy, $\mathrm{AD}$ and $\mathrm{HD}$ [8-13]. Increased uptake of ${ }^{11} \mathrm{C}$ PK11195 was also reported in frontotemporal lobar degeneration [14] and HD [15]. In preclinical HD, regional brain atrophy and increased levels of microglial activation in the associative striatum areas (AST) correlated with HD onset [13]. Early striatal dysfunction and cortical atrophy have been previously widely documented and suggested to mark the zone of onset in preclinical presymptomatic $\operatorname{HD}[16,17]$. Once extended and confirmed, these data might theoretically provide the starting point for studies validating novel premanifest biomarkers. Recently a new promising radioligand with greater affinity for TSPO than PK11195 has been developed. $\left[{ }^{18} \mathrm{~F}\right]$ FEDAA1106 has been proposed for the clinical evaluation of neuroinflammatory diseases and its biodistribution has been recently published [18].

\section{Monoamine oxidase}

Monoamine oxidase is an enzyme located at the outer mitochondrial membrane that catalyses the oxidative deamination of a wide range of monoamines, thereby influencing the concentration of neurotransmitter amines. It occurs in two subtypes, MAO-A and MAO-B, which are different gene products. These two isoenzymes differ in their substrate specificities and in their sensitivities to the inhibitors clorgiline and L-deprenyl [19].

MAO-B is localized predominantly in astrocytes and serotonergic neurons, whereas MAO-A is present in catecholaminergic neurons. MAO-B activity has been described to increase with age [20] and in brains of patients with neurodegenerative disorders such as $\mathrm{AD}$ [21], HD [22] and ALS [23] as well as after brain injury [37]. MAO-B is localized mainly in glial cells. Increased MAO-B activity may be due to astrocytic proliferation as well as an increased content of MAO-B in reactive astrocytes [24]. MAO-B levels can be estimated using L-deprenyl-D2 (DED), a selective irreversible MAO-B antagonist, which has been developed as a PET tracer [25]. Recent PET studies reported significant increase in DED binding in patients with moderate to severe $\mathrm{AD}$ compared with healthy control subjects [1]. Similar results were reported in other neurodegenerative disorders such as ALS [26] and Creutzfeldt-Jakob disease [27]. These findings suggest that in vivo imaging of activated astrocytes is a promising additional tool useful to assess prognosis and monitoring disease activity and treatment.

\section{Amyloid imaging}

Activated microglia are present at sites of aggregated $A \beta$ deposition in the brains of AD subjects, although their precise role in the disease process remains unclear [28]. The chemokine receptor CX3CR1 is selectively expressed in microglia and is thought to modulate their activity. Expression of various chemokines as well as plasma levels of fractalkine was reported to be increased in the aging brain and $\mathrm{AD}$ patients [29]. A recent study on a mouse model reported lower brain levels of $A \beta 40$ and $A \beta 42$ and reduced amyloid deposits in CX3CX1-deficient mice [30]. The authors reported that CX3CR1 deletion is associated with increased phagocytic ability, which led to greater amyloid content within microglial phagolysosomes. Pittsburgh compound B [PIB; 2-(4'-methylaminophenyl)-6-hydroxybenzothiazole or 6-OH-BTA-1] binds specifically to fibrils of $A \beta 40$ peptides and is a sensitive marker for $A \beta$ pathology in mild cognitive impairment (MCI) and $\mathrm{AD}$. PIB has provided quantitative information on $A \beta$ burden, leading to new insights into $A \beta$ deposition in neurodegenerative disease in which $A \beta$ may play a role [31]. PET studies have shown not only a significative difference in ${ }^{11} \mathrm{C}$-PIB retention between agematched healthy controls and $\mathrm{AD}$ patients [31], but also inverse correlations with glucose hypometabolism [31]. Furthermore, comparison of the diagnostic utility of $A \beta$ versus 2-[ $\left[{ }^{18} \mathrm{~F}\right]$-fluoro-2-deoxy-D-glucose $\left({ }^{18} \mathrm{~F}-\mathrm{FDG}\right)$ imaging demonstrated that PIB imaging may provide an additional tool for the diagnosis of $A D$ [32]. In dementia with Lewy bodies (DLB) patients show an ${ }^{11} \mathrm{C}$-PIB retention pattern similar to that observed in $\mathrm{AD}$ patients.

Preliminary studies report that cognitively normal elderly subjects show an increased ${ }^{11} \mathrm{C}$-PIB retention in the prefrontal and posterior cingulate/precuneus regions [31]. This increased retention is reported to be lower than that observed in $\mathrm{AD}$ subjects. The different increase in ${ }^{11} \mathrm{C}-\mathrm{PIB}$ PET retention reported in normal controls, $\mathrm{AD}$ and DLB patients reflects a broad range of $A \beta$ deposition [31] which may be related to the degree of microglia activation and/or CX3CR1 expression [30].

\section{Conclusion}

There is growing evidence that brain function is likely to reflect a complex scenario which involves pathways and multifunctional cells whose functions are strictly connected. Therefore, it is likely that not one single target or parameter will be used for assessment of neuroinflammation in the future, but a combination of parameters which will allow one to evaluate the role of inflammation in neurodegenerative disorders in its full complexity. Along with new tracers, the next future will bring us the hybrid PET/MR scanner which will improve the anatomical localization of molecular targets 
and the binding quantitation of molecular tracers through the use of a scanner native software for the correction of partial volume effects on the simultaneously acquired functional and structural images. Interesting perspectives could be also connected with an evaluation of altered pathophysiological parameters such as blood volume, perfusion and blood-brain barrier (BBB) permeability. In this field, together with a better integration between functional PET and functional MRI (or dynamic CT), a major improvement could derive from a revival of old studies allowing a quantitative BBB permeability measurement using rubidium [33] or ${ }^{68} \mathrm{Ga}$-ethylenediaminetetraacetic acid (EDTA) whose kinetics is not influenced by cellular compartment [34, 35].

Therefore, considering the availability of new tracers and imaging modalities, nuclear medicine will be able to play a relevant role for early diagnosis, selection of patients for therapies and treatment evaluation in this field [36].

\section{References}

1. Santillo AF, Gambini JP, Lannfelt L, Långström B, Ulla-Marja L, Kilander L, et al. In vivo imaging of astrocytosis in Alzheimer's disease: an (11)C-L-deuteriodeprenyl and PIB PET study. Eur J Nucl Med Mol Imaging 2011. doi:10.1007/s00259-011-1895-9.

2. Sofroniew MV. Molecular dissection of reactive astrogliosis and glial scar formation. Trends Neurosci 2009;32:638-47. doi:10.1016/ j.tins.2009.08.002.

3. Lansbury PT, Lashuel HA. A century-old debate on protein aggregation and neurodegeneration enters the clinic. Nature 2006;443:774-9. doi:10.1038/nature05290.

4. Barres BA. The mystery and magic of glia: a perspective on their roles in health and disease. Neuron 2008;60:430-40. doi:10.1016/j. neuron.2008.10.013.

5. Jamin N, Neumann JM, Ostuni MA, Vu TK, Yao ZX, Murail S, et al. Characterization of the cholesterol recognition amino acid consensus sequence of the peripheral-type benzodiazepine receptor. Mol Endocrinol 2005;19:588-94. doi:10.1210/me.2004-0308.

6. Lacapère JJ, Papadopoulos V. Peripheral-type benzodiazepine receptor: structure and function of a cholesterol-binding protein in steroid and bile acid biosynthesis. Steroids 2003;68:569-85.

7. Sutter AP, Maaser K, Höpfner M, Barthel B, Grabowski P, Faiss $\mathrm{S}$, et al. Specific ligands of the peripheral benzodiazepine receptor induce apoptosis and cell cycle arrest in human esophageal cancer cells. Int J Cancer 2002;102:318-27. doi:10.1002/ijc.10724.

8. Cagnin A, Brooks DJ, Kennedy AM, Gunn RN, Myers R, Turkheimer FE, et al. In-vivo measurement of activated microglia in dementia. Lancet 2001;358:461-7. doi:10.1016/S0140-6736 (01)05625-2.

9. Pappata S, Levasseur M, Gunn RN, Myers R, Crouzel C, Syrota A, et al. Thalamic microglial activation in ischemic stroke detected in vivo by PET and [11C]PK1195. Neurology 2000;55:1052-4.

10. Banati RB, Cagnin A, Brooks DJ, Gunn RN, Myers R, Jones T, et al. Long-term trans-synaptic glial responses in the human thalamus after peripheral nerve injury. Neuroreport 2001;12:3439-42.

11. Junck L, Olson JM, Ciliax BJ, Koeppe RA, Watkins GL, Jewett $\mathrm{DM}$, et al. PET imaging of human gliomas with ligands for the peripheral benzodiazepine binding site. Ann Neurol 1989;26:752-8. doi:10.1002/ana.410260611.
12. Goerres GW, Revesz T, Duncan J, Banati RB. Imaging cerebral vasculitis in refractory epilepsy using [(11)C](R)-PK11195 positron emission tomography. AJR Am J Roentgenol 2001;176:1016-8.

13. Politis M, Pavese N, Tai YF, Kiferle L, Mason SL, Brooks DJ, et al. Microglial activation in regions related to cognitive function predicts disease onset in Huntington's disease: a multimodal imaging study. Hum Brain Mapp 2011;32:258-70. doi:10.1002/ hbm. 21008 .

14. Cagnin A, Rossor M, Sampson EL, Mackinnon T, Banati RB. In vivo detection of microglial activation in frontotemporal dementia. Ann Neurol 2004;56:894-7. doi:10.1002/ana.20332.

15. Pavese N, Gerhard A, Tai YF, Ho AK, Turkheimer F, Barker RA, et al. Microglial activation correlates with severity in Huntington disease: a clinical and PET study. Neurology 2006;66:1638-43. doi:10.1212/01.wnl.0000222734.56412.17.

16. Ciarmiello A, Cannella M, Lastoria S, Simonelli M, Frati L, Rubinsztein DC, et al. Brain white-matter volume loss and glucose hypometabolism precede the clinical symptoms of Huntington's disease. J Nucl Med 2006;47:215-22.

17. Squitieri F, Cannella M, Simonelli M, Sassone J, Martino T, Venditti E, et al. Distinct brain volume changes correlating with clinical stage, disease progression rate, mutation size, and age at onset prediction as early biomarkers of brain atrophy in Huntington's disease. CNS Neurosci Ther 2009;15:1-11. doi:10.1111/j.1755-5949.2008.00068.x.

18. Takano A, Gulyás B, Varrone A, Karlsson P, Sjoholm N, Larsson $\mathrm{S}$, et al. Biodistribution and radiation dosimetry of the $18 \mathrm{kDa}$ translocator protein (TSPO) radioligand [(18)F]FEDAA1106: a human whole-body PET study. Eur J Nucl Med Mol Imaging 2011. doi:10.1007/s00259-011-1864-3.

19. Youdim MB, Bakhle YS. Monoamine oxidase: isoforms and inhibitors in Parkinson's disease and depressive illness. Br J Pharmacol 2006;147 Suppl 1:S287-96. doi:10.1038/sj.bjp.0706464.

20. Saura J, Andrés N, Andrade C, Ojuel J, Eriksson K, Mahy N. Biphasic and region-specific MAO-B response to aging in normal human brain. Neurobiol Aging 1997;18:497-507.

21. Jossan SS, Gillberg PG, Gottfries CG, Karlsson I, Oreland L. Monoamine oxidase B in brains from patients with Alzheimer's disease: a biochemical and autoradiographical study. Neuroscience 1991:45:1-12.

22. Mann JJ, Kaplan RD, Bird ED. Elevated postmortem monoamine oxidase B activity in the caudate nucleus in Huntington's disease compared to schizophrenics and controls. J Neural Transm 1986;65:277-83.

23. Ekblom J, Jossan SS, Oreland L, Walum E, Aquilonius SM. Reactive gliosis and monoamine oxidase B. J Neural Transm Suppl 1994;41:253-8.

24. Ekblom J, Jossan SS, Bergström M, Oreland L, Walum E, Aquilonius SM. Monoamine oxidase-B in astrocytes. Glia 1993;8:122-32. doi:10.1002/glia.440080208.

25. Fowler JS, MacGregor RR, Wolf AP, Arnett CD, Dewey SL, Schlyer $\mathrm{D}$, et al. Mapping human brain monoamine oxidase A and B with 11C-labeled suicide inactivators and PET. Science 1987;235:481-5.

26. Johansson A, Engler H, Blomquist G, Scott B, Wall A, Aquilonius SM, et al. Evidence for astrocytosis in ALS demonstrated by [11C](L)-deprenyl-D2 PET. J Neurol Sci 2007;255:17-22. doi:10.1016/j.jns.2007.01.057.

27. Engler H, Lundberg PO, Ekbom K, Nennesmo I, Nilsson A, Bergström M, et al. Multitracer study with positron emission tomography in Creutzfeldt-Jakob disease. Eur J Nucl Med Mol Imaging 2003;30:85-95. doi:10.1007/s00259-002-1008-x.

28. McGeer PL, Rogers J. Anti-inflammatory agents as a therapeutic approach to Alzheimer's disease. Neurology 1992;42:447-9.

29. Kim TS, Lim HK, Lee JY, Kim DJ, Park S, Lee C, et al. Changes in the levels of plasma soluble fractalkine in patients with mild cognitive impairment and Alzheimer's disease. Neurosci Lett 2008;436:196-200. doi:10.1016/j.neulet.2008.03.019. 
30. Liu Z, Condello C, Schain A, Harb R, Grutzendler J. CX3CR1 in microglia regulates brain amyloid deposition through selective protofibrillar amyloid-beta phagocytosis. J Neurosci 2010;30:17091-101. doi:10.1523/JNEUROSCI.4403-10.2010.

31. Rowe CC, Ng S, Ackermann U, Gong SJ, Pike K, Savage G, et al. Imaging beta-amyloid burden in aging and dementia. Neurology 2007;68:1718-25. doi:10.1212/01.wnl.0000261919.22630.ea.

32. Ng S, Villemagne VL, Berlangieri S, Lee ST, Cherk M, Gong SJ, et al. Visual assessment versus quantitative assessment of 11CPIB PET and 18F-FDG PET for detection of Alzheimer's disease. J Nucl Med 2007;48:547-52.

33. Yen CK, Yano Y, Budinger TF, Friedland RP, Derenzo SE, Huesman RH, et al. Brain tumor evaluation using $\mathrm{Rb}-82$ and positron emission tomography. J Nucl Med 1982;23:532-7.
34. Iannotti F, Fieschi C, Alfano B, Picozzi P, Mansi L, Pozzilli C, et al. Simplified, noninvasive PET measurement of blood-brain barrier permeability. J Comput Assist Tomogr 1987;11:390-7.

35. Pozzilli C, Bernardi S, Mansi L, Picozzi P, Iannotti F, Alfano B, et al. Quantitative assessment of blood-brain barrier permeability in multiple sclerosis using 68-Ga-EDTA and positron emission tomography. J Neurol Neurosurg Psychiatry 1988;51:1058-62.

36. Carrió I. Key role of nuclear medicine in bringing imaging biomarkers into clinical practice. Eur J Nucl Med Mol Imaging 2009;36:1937-9. doi:10.1007/s00259-009-1318-3.

37. Stenström A, Arai Y, Oreland L. Intra- and extraneuronal monoamineoxidase-A and -B activities after central axotomy (hemisection) on rats. J Neural Transm 1985;61:105-13. 